\title{
OPEN Leakage of astrocyte-derived extracellular vesicles in stress-induced exhaustion disorder: a cross-sectional study
}

\author{
Johanna Wallensten ${ }^{1,2 \bowtie}$, Anna Nager ${ }^{3}$, Marie Åsberg ${ }^{2}$, Kristian Borg² , Aniella Beser², \\ Alexander Wilczek ${ }^{2}$ \& Fariborz Mobarrez ${ }^{4}$
}

Patients with stress-induced exhaustion disorder (SED) demonstrate cognitive dysfunction similar to patients with minor traumatic brain injury (TBI). We have previously detected elevated concentrations of astrocyte-derived extracellular vesicles (EVs) in patients with TBI. As such, we hypothesized that astrocyte-derived EVs could be higher in patients with SED than in patients with major depressive disorder (MDD) and healthy controls. Patients with SED $(n=31)$, MDD $(n=31)$, and healthy matched controls $(n=61)$ were included. Astrocyte-derived EVs (previously known as microparticles) were measured in plasma with flow cytometry and labeled against glial fibrillary acidic protein (GFAP) and aquaporin 4 (AQP4). In addition, platelet EVs and their CD40 ligand expression were measured. Patients with SED had significantly higher concentrations of AQP4 and GFAP-positive EVs and EVs co-expressing AQP4/GFAP than patients with MDD and healthy controls. Patients with MDD had significantly higher concentrations of GFAP-positive EVs and EVs co-expressing AQP4/GFAP than healthy controls. Platelet EVs did not differ between groups. CD40 ligand expression was significantly higher in patients with SED and MDD than in controls. In conclusion, the present study suggests that patients with SED, and to some extent, patients with MDD, have increased leakage of astrocytederived EVs through the blood-brain barrier.

Prolonged, unrelieved, intensive stress can lead to a state of chronic exhaustion that is characterized by profound fatigue, emotional instability, impaired sleep, and cognitive problems such as attentional and executive dysfunction. A century ago, the condition was called acquired neurasthenia and was typically thought to be caused by overwork. More recently, it has been called burnout, but since burnout usually refers to an emotional reaction to adverse work conditions ${ }^{1}$ rather than to an illness, this label appears to be a misnomer. In an attempt to differentiate more severe clinical illness, a growing number of researchers now use the term clinical burnout ${ }^{2}$. More recently, subject experts have introduced the term stress-induced exhaustion disorder (SED) (Table 1) and provided a description of the disorder's main symptoms and course ${ }^{3}$. SED markedly impairs people's capacity to work and function in daily life and is often associated with long periods of sick leave ${ }^{4}$.

Many patients with SED are anxious and sad and display depressive symptoms ${ }^{3}$. Thus, SED is sometimes regarded as a variety of depression (e.g., exhaustion depression or job stress-induced depression). SED and

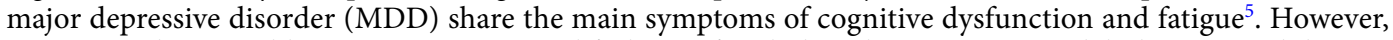
patients with SED seldom experience general feelings of guilt, hopelessness, or suicidal ideation, and depressive symptoms are not their main complaint ${ }^{6}$. Moreover, the natural course and treatment outcome of SED and MDD appear to differ ${ }^{7,8}$.

The main symptoms of SED are similar to symptoms of minor traumatic brain injury (TBI), i.e., post-concussion syndrome. The cognitive impairments that patients with SED experience, such as impaired attention, executive function, and memory, may last for several months or even years ${ }^{9}$. Research has demonstrated structural changes in the medial prefrontal cortex, hippocampus, and amygdala of patients with $\mathrm{SED}^{10}$. However, an imaging study has found that after 1-2 years, the thinned prefrontal cortex of patients with SED returns to

\footnotetext{
${ }^{1}$ Academic Primary Health Care Centre, Region Stockholm, Solnavägen 1E, Box 45436, 10431 Stockholm, Sweden. ${ }^{2}$ Department of Clinical Sciences, Karolinska Institutet, Danderyd University Hospital, 18288 Stockholm, Sweden. ${ }^{3}$ Division of Family Medicine and Primary Health Care, Department of Neurobiology, Care Sciences and Society, Karolinska Institutet, 14152 Stockholm, Sweden. ${ }^{4}$ Department of Medical Sciences, Uppsala University, 75185 Uppsala, Sweden. ${ }^{\circledR}$ email: johanna.wallensten@ki.se
} 


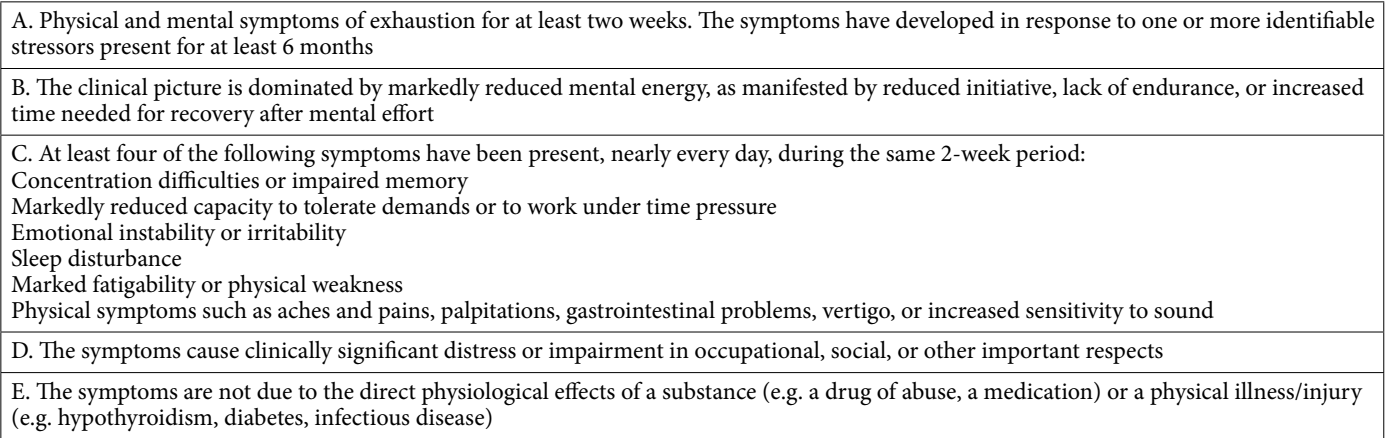

Table 1. Criteria for stress-induced exhaustion disorder according to the Swedish National Board of Health and Welfare and Swedish version of the International Classification of Diseases, 10th edition (code F-43.8). Criteria A through E must be fulfilled to diagnose stress-induced exhaustion disorder.

normal $^{11}$. Structural brain changes in patients with SED are similar to those found in patients with early life traumas ${ }^{12}$ or post-traumatic stress disorder ${ }^{13}$ and to those seen in rats subjected to chronic stress ${ }^{14}$.

In several neurological disorders, such as TBI and neurodegenerative disorders, increased concentrations of extracellular vesicles (EVs) from astrocytes have been detected in peripheral blood or cerebrospinal fluid ${ }^{15,16}$. EVs, also known as microparticles, are released during cell activation and/or apoptosis and resemble their parent cells in that they expose similar cell-specific and activation markers ${ }^{17}$. Astrocytes, which are important regulators in the formation and maintenance of the blood-brain barrier $(\mathrm{BBB})^{18}$, can also release EVs upon stimulation. In a previous study of patients with TBI, we found elevated concentrations of astrocyte-derived EVs expressing glial fibrillary acidic protein (GFAP) and water channel protein aquaporin 4 (AQP4) ${ }^{16}$. In light of the symptoms of brain dysfunction and structural changes in the brain in patients with SED, as well as our findings of astrocytederived EVs in patients with TBI, we hypothesized that concentrations of EVs of astrocytic origin are increased in the peripheral blood of patients with SED. We therefore examined whether astrocyte-derived EVs are found in the peripheral blood of patients with SED, and if so, whether concentrations of these EVs differ in patients with SED, patients with MDD, and healthy controls.

\section{Methods}

Study design and participants. The study included 31 patients with SED, 31 patients with MDD, and 61 healthy controls. Patients with SED and patients with MDD present overlapping symptoms, and patients with MDD were therefore used as clinical controls. Between 2016 and 2018, patients with SED or MDD treated at a psychiatric outpatient clinic in Stockholm were consecutively included in the study. The clinic specialized in treating health care professionals with symptoms of SED, depression, and anxiety. Since too few patients with MDD could be recruited from the clinic, additional patients with MDD were recruited from a public outpatient clinic in Stockholm in 2018.

Patients who were diagnosed with SED or MDD were asked to participate in the study by their physician, occupational therapist, or nurse. Inclusion criteria were ongoing SED (Table 1) or MDD, age 18 through 65 years, ability to understand Swedish, willingness to undergo 30 to $40 \mathrm{~min}$ of standardized testing, and diagnosis no more than three months prior to inclusion. Patients fulfilling diagnostic criteria for both SED and MDD were included in the study only if the physician considered the patient's depressive symptoms secondary to the symptoms of SED, and in that case in the SED group. Patients with somatic or psychiatric diagnoses that could explain their symptoms better than SED or MDD were not included.

Physicians at the clinics diagnosed the patients with SED or MDD. The Mini-International Neuropsychiatric Interview (M.I.N.I) was used to exclude other psychiatric diagnoses. M.I.N.I was administered by a professional familiar with the instrument, either a clinic physician, clinic psychologist, or one of the researchers. Blood samples were obtained to exclude subclinical thyroid disease, anemia, vitamin B12 deficiency, or alcohol overconsumption.

Healthy controls were recruited in 2009 to provide blood samples to serve as reference material for future studies. At the request of the research group, Statistics Sweden, Sweden's official government statistics agency, randomly selected 1146 permanent residents in the Stockholm area, aged 28 through 55, from the Swedish population register. The research group sent invitation letters to these people, and those who agreed to participate were contacted by phone for a first screening. People who reported that they were healthy were invited to an investigation that included a clinical and psychological examination. The investigation was performed by a physician. Those with current or previous SED, any other mental illness, endocrine or cardiovascular disorder, or cancer were excluded, resulting in 165 healthy controls who provided blood samples. Sixty-one of these 165 healthy controls, matched as closely as possible in age and sex to the SED and MDD groups, were selected for analysis of EVs.

The study was approved by the regional Ethical Review Board in Stockholm, Sweden (http://www.epn.se/ en/start/) (Dnr. 2014/585-31/1, 2016/1239-32 and 2017/970-21/1). All participants received verbal and written 
A

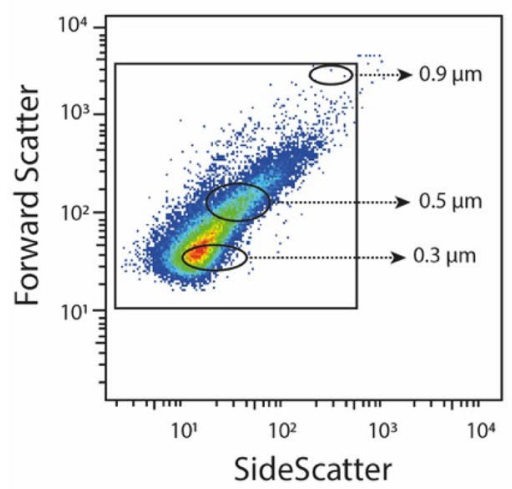

B

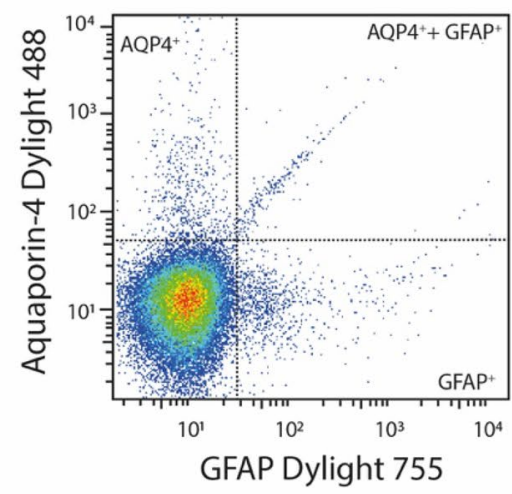

C

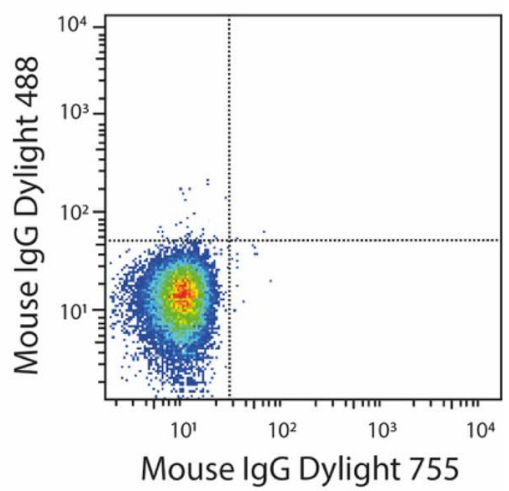

Figure 1. Flow cytometric analysis of astrocyte-specific extracellular vesicles in patients with stress-induced exhaustion disorder (SED). (A) Representative dot-plot of extracellular vesicle gating (based on size and complexity) together with gates demonstrating beads with a diameter of $0.3,0.5$, and $0.9 \mu \mathrm{m}$. (B) Representative dot-plot demonstrating aquaporin 4 (AQP4) and glial fibrillary acidic protein (GFAP) expression as well as vesicles exposing both AQP4 and GFAP. (C) Representative dot-plot demonstrating isotype mouse controls.

information about the study and provided written informed consent in accordance with the Declaration of Helsinki. All data were pseudonymized before linkage and analysis.

Symptom rating scales. The self-assessment version of the Montgomery Asberg Depression Rating Scale (MADRS-S) ${ }^{19}$ was used to quantify the severity of depressive symptoms. It includes nine items rated on a scale from 0 to 6; higher scores indicate more severe symptoms. The 25-item Cognitive Failures Questionnaire (CFQ) was used to measure experience of cognitive failures in daily life, including failures of perception, memory, and motor function. Response alternatives range from 0 (never) to 4 (very often); higher scores indicate more frequent problems ${ }^{20}$.

Sample collection. At inclusion, blood samples were obtained from patients with SED and MDD in accordance with a standardized protocol. All patients were asked to refrain from alcohol and physical exertion prior to blood sampling and to abstain from leaving a sample if they had signs of ongoing infection. Blood samples were drawn with a $21 \mathrm{G}$ sampling needle in the morning after overnight fasting, through direct venepuncture from an antecubital vein, after at least $15 \mathrm{~min}$ of rest. Samples were drawn into citrated tubes and centrifuged within $1 \mathrm{~h}$ at $2000 \mathrm{~g}$ for $20 \mathrm{~min}$ at room temperature (RT), and subsequently stored at $-80^{\circ} \mathrm{C}$ as platelet poor plasma. Blood samples for patients and controls were analyzed at Danderyd Hospital, Stockholm.

Flow cytometric measurement of extracellular vesicles. Samples were thawed in a water bath at $37^{\circ} \mathrm{C}$ for $5 \mathrm{~min}$ and subsequently centrifuged at $2000 \mathrm{~g}$ for $20 \mathrm{~min}$ at RT. The upper supernatant was transferred to new tubes and centrifuged at $20800 \mathrm{~g}$ for $45 \mathrm{~min}$ at RT. The supernatant obtained by this second centrifugation step was discarded, and the EV-enriched pellet was used for the flow cytometric analysis. Subsequently, $20 \mu \mathrm{L}$ of the pellet were incubated for $20 \mathrm{~min}$ in darkness, with $5 \mu \mathrm{L}$ of anti-Aquaporin- 4 Dylight 488 (corresponding to Human Aquaporin 4 aa 50-150, Abcam, Cambridge, UK), $5 \mu \mathrm{L}$ anti-GFAP Dylight 755 (Abcam, Cambridge, $\mathrm{UK}$ ), $5 \mu \mathrm{L}$ CD41-FITC (Beckman coulter, Brea, CA, USA), and/or $5 \mu \mathrm{L}$ CD154-PE (Beckman coulter, Brea, CA, USA). EVs were measured by flow cytometry on a Beckman Gallios instrument (Beckman coulter, Brea, CA, USA) with the threshold set to forward scatter. The EV gate was determined using Megamix-Plus FSC beads $(0 \cdot 3$, 0.5 and $0.9 \mu \mathrm{m}$ in size; BioCytex, Marseille, France). EVs were defined as vesicles less than $0.9 \mu \mathrm{m}$ in diameter (forward scatter) and positive for the antibodies described above.

For the sake of brevity and convenience, in this study, microvesicles defined by their size and expression of GFAP and AQP4 (both single- and double-positive events were measured) are referred to as astrocyte-derived EVs. Both GFAP and AQP4 are markers of astrocytes ${ }^{16}$. AQP4 is a bidirectional water channel that is mainly found on astrocytes throughout the central nervous system ${ }^{21}$. However, GFAP has also been found in cells outside the central nervous system, such as lymphocytes ${ }^{22}$ and fibroblasts ${ }^{23}$.

In addition to measuring astrocyte-derived EVs, we also measured the concentration of platelet-derived EVs (CD41) and their expression of CD154 (CD40 Ligand). Conjugate isotype-matched immunoglobulin with no reactivity against human antigens was used as a negative control to define the background noise in the cytometric analysis (Fig. 1). Results are presented as EVs/ $\mu \mathrm{L}$ plasma, processed from the $20 \mu \mathrm{L}$ pellet obtained after highspeed centrifugation. The intra- and interassay coefficients of the flow cytometric analysis, which was performed with 10 normal pool plasma samples ( 5 intra- and 5 interassays), were each less than $9.0 \%$.

Statistical methods. Non-parametric or parametric tests were used as appropriate to compare descriptive characteristics of groups. The Kruskal-Wallis test was used for non-parametric tests of three groups and the Mann-Whitney test for two groups. ANOVA was used for parametric tests of three groups and un-paired t-tests 


\begin{tabular}{|c|c|c|c|c|}
\hline Descriptive characteristics & $\begin{array}{l}\text { Stress-induced } \\
\text { exhaustion disorder (SED) }\end{array}$ & $\begin{array}{l}\text { Major depressive disorder } \\
\text { (MDD) }\end{array}$ & Healthy controls & $P$ value \\
\hline Mean age in years & $44 \cdot 6(9 \cdot 7) \quad \mathrm{n}=31$ & $40 \cdot 3(10 \cdot 8) \quad n=31$ & $42 \cdot 2(9 \cdot 5) \quad \mathrm{n}=61$ & 0.206 \\
\hline Women & $\mathrm{n}=31$ & $\mathrm{n}=31$ & $52(85 \cdot 2 \%) \quad n=61$ & 0.999 \\
\hline Mean BMI & $\mathrm{n}=31$ & $\mathrm{n}=31$ & $24.7(3.6) \quad \mathrm{n}=61$ & 0.898 \\
\hline Mean MADRS-S sum & $\mathrm{n}=22$ & $\mathrm{n}=25$ & $\mathrm{n}=61$ & $0.001^{*}$ \\
\hline Mean CFQ sum & $\mathrm{n}=14$ & $50 \cdot 0(12 \cdot 1) \quad \mathrm{n}=26$ & $\mathrm{n}=0$ & $0.025^{* *}$ \\
\hline $\begin{array}{l}\text { Mean leukocyte concentration } \\
\left(10^{9} / \mathrm{L}\right)\end{array}$ & $\mathrm{n}=28$ & $\mathrm{n}=28$ & $\mathrm{n}=61$ & $0.023^{* * *}$ \\
\hline $\begin{array}{l}\text { Mean erythrocyte concentration } \\
\left(10^{12} / \mathrm{L}\right)\end{array}$ & $\mathrm{n}=28$ & $4 \cdot 6(0.4)$ & $\mathrm{n}=0$ & 0.610 \\
\hline $\begin{array}{l}\text { Mean platelet concentration } \\
\left(10^{9} / \mathrm{L}\right)\end{array}$ & $271.1(92.0)$ & $251.8(49.5)$ & $218.6(26 \cdot 4) \quad n=61$ & $0.002^{*}$ \\
\hline
\end{tabular}

Table 2. Descriptive characteristics of patients with stress-induced exhaustion disorder $(\mathrm{n}=31)$, patients with major depressive disorder $(n=31)$, and healthy controls $(n=61)$. Data are mean $(S D)$ or $n(\%)$. ${ }^{*}$ Significant at a 0.05 level between SED and healthy controls and between MDD and healthy controls. ${ }^{*}$ Significant at a 0.05 level between SED and MDD. ${ }^{* *}$ Significant at a 0.05 level between MDD and healthy controls.

for parametric tests of two groups. Bonferroni was used as a post-hoc test of Kruskal-Wallis and ANOVA tests. Prior to statistical analysis, EV data were log transformed if necessary to obtain a normal distribution. Patients and controls were compared with the un-paired t-test after normalization. $P$ values of $\leq 0.05$ were considered significant. Statistical analysis was performed using SPSS Statistics (IBM SPSS Statistics for Windows, v 26.0. Armonk, NY: IBM Corp.) and JMP software (SAS Institute, v12.0, Cary, North Carolina, USA).

\section{Results}

Descriptive characteristics. A total of 31 patients with SED, 31 patients with MDD, and 61 healthy controls were recruited to the study. None were excluded because of subclinical thyroid disease, anemia, vitamin B12 deficiency, or alcohol overconsumption. There were no significant differences between the three groups in age, sex, BMI, or erythrocyte levels (Table 2). MADRS-S scores differed significantly between the groups in a manner consistent with the diagnoses $(P=0 \cdot 001)$. That is, patients with MDD had the highest MADRS-S scores, and patients with SED had MADRS-S scores lower than those with MDD but higher than those of healthy controls. CFQ scores also differed significantly between patients with SED and patients with MDD $(P=0.025)$; the highest scores were observed in patients with SED. Blood sample concentrations of leukocytes, erythrocytes, and platelets were normal in all groups except for one outlier in the SED group who had an elevated platelet concentration $\left(657 \times 10^{9} / \mathrm{L}\right)$. Leukocyte concentration differed significantly between the three groups $(P=0.023)$, with higher levels in patients with MDD than in healthy controls. Platelet concentrations differed significantly between the three groups $(P=0.002)$, with higher concentrations in patients with either SED or MDD than in healthy controls.

All patients with MDD and no healthy controls received antidepressant medication. There was no significant difference in EV concentration between patients with SED who received antidepressant medication $(n=5)$ and patients with SED who did not receive such medication $(n=26)$ (data not shown). In addition, there were no significant differences in the concentrations of leucocytes, erythrocytes, or platelets between patients with SED who received antidepressant medication $(n=5)$ and patients with SED who did not receive such medication $(n=26)$.

Extracellular vesicles. To detect astrocyte-derived EVs, we labeled the EVs with two astrocyte-specific markers, AQP4 and GFAP. Patients with SED had significantly higher concentrations of AQP4-positive EVs than patients with MDD $(P<0.001)$ and controls $(P<0.01)$ (Fig. $2 \mathrm{~A})$. GFAP-positive EVs followed the same pattern: higher concentration of GFAP-positive EVs were observed in patients with SED than either patients with MDD $(P<0.001)$ or healthy controls $(P<0.01)$ (Fig. 2B). We also investigated EVs that co-expressed AQP4 and GFAP, because such co-expression may provide a stronger indication that the EVs originate from astrocytes (Fig. 2C). Concentrations of EVs co-expressing AQP4 and GFAP were significantly higher in patients with SED than either patients with MDD $(P<0.001)$ or healthy controls $(P<0.01)$. Patients with MDD had significantly higher concentrations of both GFAP $(P<0 \cdot 05)$ and EVs co-expressing AQP4 and GFAP $(P<0 \cdot 001)$ than healthy controls.

In addition to astrocyte-derived EVs, we also investigated EVs originating from platelets $\left(\mathrm{CD} 41^{+}\right)$and EVs expressing the inflammation marker CD154 (CD40 Ligand). There were no significant differences between groups in concentration of CD41-positive EVs (Fig. 3A). However, concentrations of EVs positive for CD40 Ligand were significantly higher in patients with SED and in patients with MDD than in healthy controls $(P<0.05)$ (Fig. 3B). No differences in CD40 Ligand expression were observed between patients with SED and patients with MDD (Fig. 3A,B). There was no correlation of clinical interest between concentrations of astrocyte-derived EVs and EVs positive for CD40 Ligand. 
A

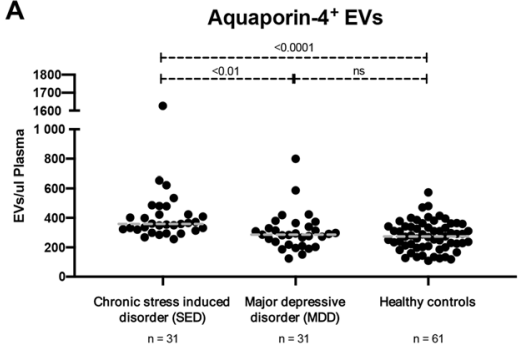

B

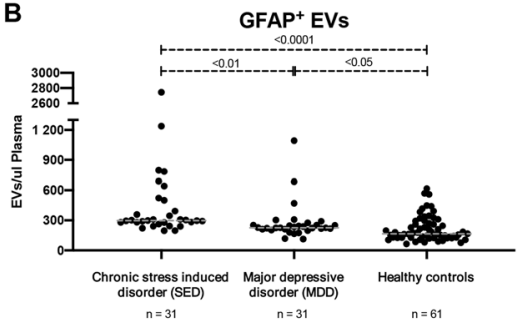

C

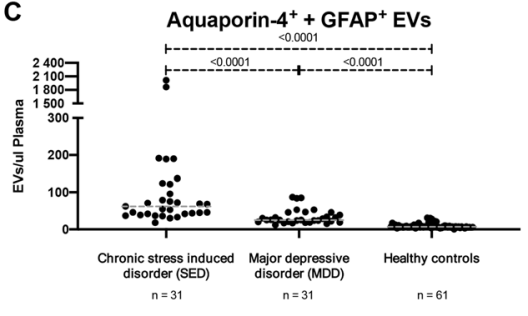

Figure 2. (A-C) Astrocyte-derived extracellular vesicles (EVs) expressing aquaporin 4 (AQP4) (A), glial fibrillary acidic protein (GFAP) (B), or both AQP4 and GFAP (C) in patients with stress-induced exhaustion disorder, patients with major depressive disorder, and healthy controls.

A

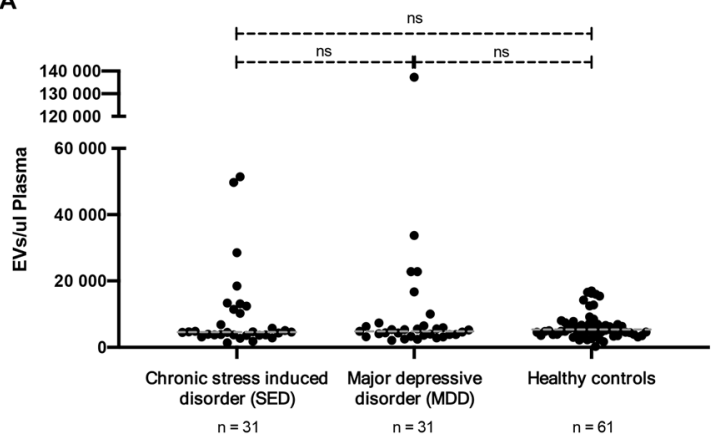

B

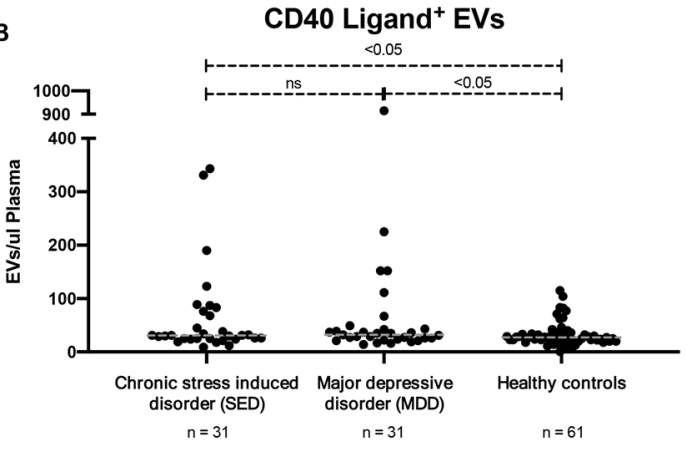

Figure 3. A and B. Extracellular vesicles (EVs) expressing platelet-specific marker CD41 (A) or CD40 Ligand (B) in patients with stress-induced exhaustion disorder, patients with major depressive disorder, and healthy controls.

\section{Discussion}

Main findings. In this study, we observed EVs of astrocyte origin in the peripheral blood of patients with SED. Patients with SED had significantly higher concentration of astrocyte-derived EVs in their peripheral blood than patients with MDD and healthy controls. Thus, release and leakage of astrocyte-derived EVs through the blood-brain barrier resembles that observed after mechanical brain damage.

Aquaporin-4 and glial fibrillary acidic protein. In the present study, we labeled astrocyte-derived EVs with two markers that can be elevated in patients with $\mathrm{TBI}^{16}$. One of these, AQP4, is a protein important for water and potassium homeostasis. It is predominantly found in the plasma membrane of astrocytes, concentrated in those domains facing vessels and pia mater, mediating the exchange of water between the brain and extracerebral liquids ${ }^{21}$. Changes in AQP4 expression and/or distribution have been found in neurological disorders such as amyotrophic lateral sclerosis, multiple sclerosis, and epilepsy, as well as in Alzheimer's disease and in TBI ${ }^{24}$.

GFAP is a protein predominantly expressed by mature and reactive astrocytes. It appears to be an indicator of TBI; its plasma concentration has been correlated with intracranial trauma visible on CT scans and with CTnegative but MRI-positive findings ${ }^{25}$. Increased expression of GFAP has also been observed in the cerebrospinal fluid and in certain brain areas of people with neurodegenerative disorders, stroke, and malignant brain tumors ${ }^{26}$.

We also investigated platelet-derived EV concentrations and EVs expressing the inflammatory marker CD40 Ligand to observe potential changes in hemostatic and inflammatory parameters. Platelets are a major source of EVs in blood and are important in the development of inflammation ${ }^{27}$. We did not detect any increase in plateletderived EVs, which suggests that release of astrocyte-derived EVs in patients with SED occurs independently of EVs that originate in blood. However, we observed slightly higher concentration of EVs that were positive for CD40 Ligand in patients with SED and MDD than in healthy controls. CD40 Ligand is released by both platelets and T-lymphocytes ${ }^{28}$. Further studies with larger sample sizes are needed to more fully investigate the role of T-lymphocytes in both SED and MDD.

Astrocyte activation. Following mechanical brain damage, astrocytes activate to protect the structure and function of neurons and to demarcate brain tissue injuries. However, if not resolved in time, astrocyte activation and formation of glial scars may reduce neuroplasticity, and slow regeneration may negatively affect neuroplasticity and regeneration in the central nervous system $(\mathrm{CNS})^{29}$.

Previous studies in rodents and postmortem studies in humans suggest brain region-specific alterations in astrocyte density and morphology in mood and stress disorders ${ }^{30}$. These changes could possibly lead to EV formation and release, as both cell activation and cell death lead to EV formation ${ }^{17}$. 


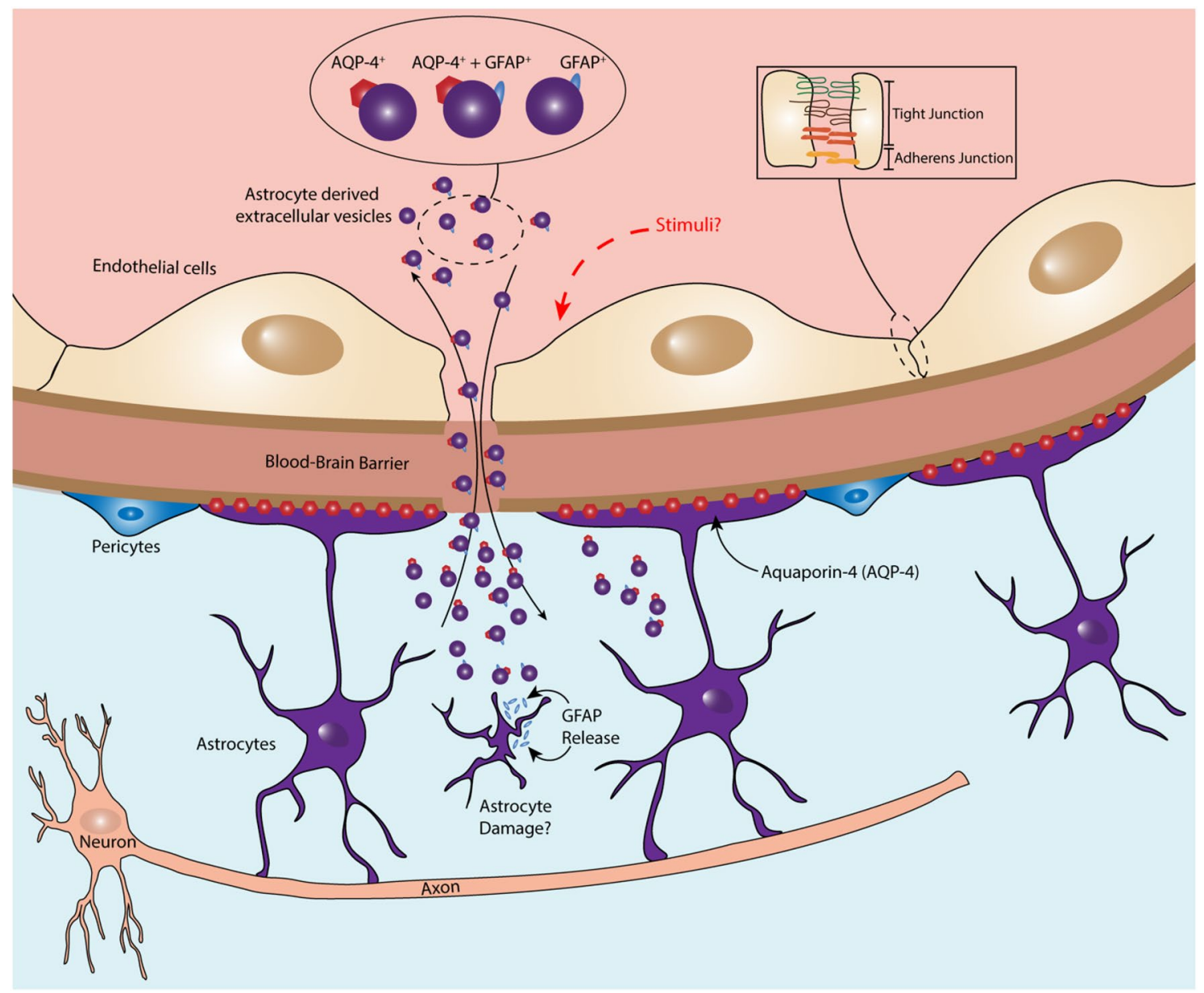

Figure 4. Our hypothesis is that stimuli can influence the blood-brain barrier to open the tight junction and adherent junction proteins, subsequently influencing astrocytes and leading to activation or apoptosis. The cellular changes cause the formation and release of extracellular vesicles, which could pass through the bloodbrain barrier and be detectable in the blood.

Our finding of astrocyte-derived EVs in peripheral blood is in line with the findings of a 2019 study that detected GFAP and astrocytic protein aldolase $\mathrm{C}$ in the serum of rats exposed to stress ${ }^{31}$. Testing indicated that the aldolase $\mathrm{C}$ derived from the brain of the rats ${ }^{31}$.

Blood-brain barrier permeability. The BBB protects the microenvironment of the CNS by restricting and controlling the passage of substances between the peripheral vascular system and the CNS. Astrocytes interact with endothelial cells of the BBB through astrocyte endfeet. In disorders characterized by mechanical CNS damage, such as brain injury and neurodegenerative disorders, it seems logical that leakage of astrocyte-derived EVs through the BBB could be measured in peripheral blood. However, the pathophysiological mechanism and thus the clinical relevance is yet to be discovered in patients with stress and mood disorders. Perhaps increased concentrations of astrocyte-derived EVs in peripheral blood, as seen in the present study and in the 2019 study of stress-exposed rats, result from increased permeability of the BBB. In stress and mood disorders, increased $\mathrm{BBB}$ permeability may enhance signaling in order to change behavior in the individual that supports recovery such as actions to decrease stress factors and increase time for rest.

Our hypothesis (Fig. 4) is that stimuli can influence the BBB to open the tight junction and adherent junction proteins. This could subsequently influence astrocytes, and depending on the type of stimuli, lead to activation or apoptosis. The cellular changes cause the formation and release of EVs, which could pass through the BBB and be detectable in the blood. The stimuli could be stress hormones, cytokines as part of inflammatory processes, and/or EVs from peripheral blood ${ }^{32}$. A study of children with obstructive sleep apnea found that EVs in peripheral blood disrupted the integrity of the BBB, especially in those with neurocognitive deficits ${ }^{32}$. Further studies are needed to better understand the processes that allow release of astrocyte-derived EVs from the CNS into peripheral blood after exposure to stress and the clinical relevance of this.

Astrocyte-derived extracellular vesicles as biomarkers of stress-related disorders. We hypothesize that the increased plasma levels of astrocyte-derived EVs reflect stress-dependent structural changes in the brain occurring during, and possibly some time after, exposure to long-term stress without sufficient recovery 
and rest. These structural changes, and subsequently the release of astrocyte-derived EVs, are probably more pronounced in patients with SED than in patients with MDD since the symptoms in SED by definition are developed in response to stressors present for at least 6 months. MDD may also develop in response to stress, and being depressed is often stressful, however, MDD is not necessarily associated with long-term stress.

As such, the structural changes in the brain are probably associated with long-term cognitive impairment in patients with SED and may not be affected by antidepressant medication. Use of antidepressant medication did not predict the course of burnout symptoms in a study on patients with SED attending a stress clinic ${ }^{6}$.

The present study suggests that it is possible to detect these stress-dependent structural changes in the brain by measuring astrocyte-derived EVs in the peripheral blood. In order to use astrocyte-derived EVs as biomarkers, the finding needs to be replicated in larger studies, as well as patients in different stages of SED and with different mood and stress disorders. In the future, astrocyte-derived EVs might be useful as biomarkers for differential diagnosis and for measuring treatment effects in patients with stress-related disorders.

\section{Limitations}

This study had several limitations. We collected blood samples from the patients during a different period than we collected them from the controls. However, blood sampling routines were the same in patients and controls, and the samples were analyzed in the same batches. AQP4 and GFAP are markers of astrocytes, but GFAP has also been found outside the central nervous system. It is therefore possible that EVs in the peripheral blood could have picked up GFAP from outside the central nervous system. We attempted to minimize this possibility by measuring for simultaneous presence of AQP4 and GFAP in the peripheral blood in all three groups. Further studies are needed to investigate the origin of the simultaneous presence of AQP4 and GFAP in peripheral blood in more detail. Furthermore, although the GFAP antigen we used is known to bind to GFAP protein, we cannot exclude the possibility that the antibody may have bound to something other than GFAP in our experiments. If unspecific binding were an issue, the concentration in healthy controls should have been higher as well, and it was not. From our study, it is clear that patients with SED and MDD have higher levels of AQP4 and GFAP exposed on EVs than healthy controls. Moreover, patients with SED and patients with MDD may have been in different stages of the diseases. To minimize this diversity, to be included in the study, patients with SED and patients with MDD had to be diagnosed less than 3 months before blood sampling, i.e., in a relatively early stage of the disorder. Lastly, the cross-sectional design makes it impossible to infer causality.

\section{Conclusions}

In conclusion, patients with SED develop clinical features resembling brain dysfunction that are similar to clinical features in patients with minor traumatic brain injury. The present data demonstrate that patients with SED have higher concentrations of astrocyte-derived EVs in their peripheral blood than patients with MDD and healthy controls. Moreover, some subtypes of astrocyte-derived EVs are higher in both patients with SED and patients with MDD than in controls. The data therefore suggest that patients with SED, and to some extent, patients with MDD, have increased leakage of astrocyte-derived EVs through the BBB. Further studies are needed to investigate this mechanism in more detail as well as the potential to use astrocyte-derived EVs in diagnosing stress-related and mood disorders and in measuring treatment effects.

\section{Data availability}

The dataset generated and analyzed during the study is available from the corresponding author on reasonable request.

Received: 4 March 2020; Accepted: 5 January 2021

Published online: 21 January 2021

\section{References}

1. Schaufeli, W. \& Enzmann, D. The Burnout Companion to Study and Practice: A Critical Analysis (Taylor and Francis, London, 1998).

2. Grossi, G., Perski, A., Osika, W. \& Savic, I. Stress-related exhaustion disorder-clinical manifestation of burnout? A review of assessment methods, sleep impairments, cognitive disturbances, and neuro-biological and physiological changes in clinical burnout. Scand. J. Psychol. 56, 626-636. https://doi.org/10.1111/sjop.12251 (2015).

3. Åsberg M, G. K., Herlofson J, Jacobsson L, Krakau I, Nygren A, Perski A, Svensson A. Utmattningssyndrom- en kunskapsöversikt om stressrelaterad psykisk ohälsa. (2003).

4. Lidwall, U. \& Olsson-Bohlin, C. Psykiatriska diagnoser. Korta analyser 2017:1. Lång väg tillbaka till arbete vid sjukskrivning. Report No. ISBN 978-91-7500-398-6 FK-nr: 116-6, (2017).

5. Knight, M. J. \& Baune, B. T. Cognitive dysfunction in major depressive disorder. Curr. Opin. Psychiatry 31, 26-31. https://doi.org/ 10.1097/yco.0000000000000378 (2018).

6. Glise, K., Ahlborg, G. Jr. \& Jonsdottir, I. H. Course of mental symptoms in patients with stress-related exhaustion: Does sex or age make a difference?. BMC Psychiatry 12, 18. https://doi.org/10.1186/1471-244X-12-18 (2012).

7. Wallensten, J., Asberg, M., Wiklander, M. \& Nager, A. Role of rehabilitation in chronic stress induced exhaustion disorder: A narrative review. J. Rehabil. Med. https://doi.org/10.2340/16501977-2545 (2019).

8. Bryngelson, A. et al. Self-reported treatment, workplace-oriented rehabilitation, change of occupation and subsequent sickness absence and disability pension among employees long-term sick-listed for psychiatric disorders: A prospective cohort study. $B M J$ Open https://doi.org/10.1136/bmjopen-2012-001704 (2012).

9. Eskildsen, A., Andersen, L. P., Pedersen, A. D. \& Andersen, J. H. Cognitive impairments in former patients with work-related stress complaints - one year later. Stress https://doi.org/10.1080/10253890.2016.1222370 (2016).

10. Blix, E., Perski, A., Berglund, H. \& Savic, I. Long-term occupational stress is associated with regional reductions in brain tissue volumes. PLoS ONE 8, e64065. https://doi.org/10.1371/journal.pone.0064065 (2013).

11. Savic, I., Perski, A. \& Osika, W. MRI shows that exhaustion syndrome due to chronic occupational stress is associated with partially reversible cerebral changes. Cereb. Cortex https://doi.org/10.1093/cercor/bhw413 (2017). 
12. van Harmelen, A. L. et al. Reduced medial prefrontal cortex volume in adults reporting childhood emotional maltreatment. Biol. Psychiatry 68, 832-838. https://doi.org/10.1016/j.biopsych.2010.06.011 (2010).

13. Pavlisa, G., Papa, J., Pavic, L. \& Pavlisa, G. Bilateral MR volumetry of the amygdala in chronic PTSD patients. Collegium Antropol. 30, 565-568 (2006).

14. Khan, A. R. et al. Biophysical modeling of high field diffusion MRI demonstrates micro-structural aberration in chronic mild stress rat brain. NeuroImage 142, 421-430. https://doi.org/10.1016/j.neuroimage.2016.07.001 (2016).

15. Bejerot, S. et al. Neuromyelitis optica spectrum disorder with increased aquaporin-4 microparticles prior to autoantibodies in cerebrospinal fluid: A case report. J. Med. Case Rep. 13, 27. https://doi.org/10.1186/s13256-018-1929-z (2019).

16. Nekludov, M., Bellander, B. M., Gryth, D., Wallen, H. \& Mobarrez, F. Brain-derived microparticles in patients with severe isolated TBI. Brain Inj. 31, 1856-1862. https://doi.org/10.1080/02699052.2017.1358395 (2017).

17. Mobarrez, F., Svenungsson, E. \& Pisetsky, D. S. Microparticles as autoantigens in systemic lupus erythematosus. Eur. J. Clin. Invest. 48, e13010. https://doi.org/10.1111/eci.13010 (2018).

18. Sofroniew, M. V. \& Vinters, H. V. J. A. Astrocytes: Biology and pathology. Acta Neuropathol. 119, 7-35. https://doi.org/10.1007/ s00401-009-0619-8 (2010).

19. Svanborg, P. \& Asberg, M. A new self-rating scale for depression and anxiety states based on the comprehensive psychopathological rating scale. Acta Psychiatr. Scand. 89, 21-28. https://doi.org/10.1111/j.1600-0447.1994.tb01480.x (1994).

20. Broadbent, D. E., Cooper, P. F., FitzGerald, P. \& Parkes, K. R. The cognitive failures questionnaire (CFQ) and its correlates. Br. J. Clin. Psychol. 21(Pt 1), 1-16. https://doi.org/10.1111/j.2044-8260.1982.tb01421.x (1982).

21. Amiry-Moghaddam, M. \& Ottersen, O. P. The molecular basis of water transport in the brain. Nat. Rev. Neurosci. 4, 991-1001. https://doi.org/10.1038/nrn1252 (2003).

22. Riol, H., Tardy, M., Rolland, B., Lévesque, G. \& Murthy, M. R. Detection of the peripheral nervous system (PNS)-type glial fibrillary acidic protein (GFAP) and its mRNA in human lymphocytes. J. Neurosci. Res. 48, 53-62 (1997).

23. Hainfellner, J. A. et al. Fibroblasts can express glial fibrillary acidic protein (GFAP) in vivo. J. Neuropathol. Exp. Neurol. 60, 449-461. https://doi.org/10.1093/jnen/60.5.449 (2001).

24. Hubbard, J. A., Szu, J. I. \& Binder, D. K. The role of aquaporin-4 in synaptic plasticity, memory and disease. Brain Res. Bull. 136, 118-129. https://doi.org/10.1016/j.brainresbull.2017.02.011 (2018).

25. Yue, J. K. et al. Association between plasma GFAP concentrations and MRI abnormalities in patients with CT-negative traumatic brain injury in the TRACK-TBI cohort: a prospective multicentre study. Lancet. Neurol. 18, 953-961. https://doi.org/10.1016/ s1474-4422(19)30282-0 (2019).

26. Middeldorp, J. \& Hol, E. M. GFAP in health and disease. Prog. Neurobiol. 93, 421-443. https://doi.org/10.1016/j.pneurobio.2011. 01.005 (2011).

27. Vajen, T., Mause, S. F. \& Koenen, R. R. Microvesicles from platelets: Novel drivers of vascular inflammation. Thromb. Haemost. 114, 228-236. https://doi.org/10.1160/TH14-11-0962 (2015).

28. Aloui, C. et al. The signaling role of CD40 ligand in platelet biology and in platelet component transfusion. Int. J. Mol. Sci. 15, 22342-22364. https://doi.org/10.3390/ijms151222342 (2014).

29. Pekny, M., Wilhelmsson, U. \& Pekna, M. The dual role of astrocyte activation and reactive gliosis. Neurosci. Lett. 565, 30-38. https://doi.org/10.1016/j.neulet.2013.12.071 (2014).

30. Murphy-Royal, C., Gordon, G. R. \& Bains, J. S. Stress-induced structural and functional modifications of astrocytes-Further implicating glia in the central response to stress. Glia https://doi.org/10.1002/glia.23610 (2019).

31. Gomez-Molina, C. et al. Small extracellular vesicles in rat serum contain astrocyte-derived protein biomarkers of repetitive stress. Int. J. Neuropsychopharmacol. 22, 232-246. https://doi.org/10.1093/ijnp/pyy098 (2019).

32. Khalyfa, A., Gozal, D. \& Kheirandish-Gozal, L. Plasma extracellular vesicles in children with OSA disrupt blood-brain barrier integrity and endothelial cell wound healing in vitro. Int. J. Mol. Sci. https://doi.org/10.3390/ijms20246233 (2019).

\section{Acknowledgements}

This study was supported by grants provided by Skandia; Söderström Königska Foundation; The Foundation for Rehabilitation and Medical Research; Karolinska Institutet Kristian Borg grants; the NVS project, Region Stockholm; and the Department of Rehabilitation Medicine, Danderyd University Hospital, Region Stockholm. Medical editor and writer Kimberly Kane, Region Stockholm, provided suggestions for editing the language in the final draft of the manuscript. The authors thank all the participants who took part in this study.

\section{Author contributions}

J.W., A.N., M.A., K.B., F.M., and A.W. conceived and designed the study. J.W., A.B., and A.W. acquired the data. J.W., A.N., F.M., and A.B. analyzed and interpreted the data. J.W., A.N., F.M., and M.Å. drafted the manuscript. All authors revised the manuscript for important intellectual content, approved the final version for publication, and agree to be accountable for all aspects of the work in ensuring that questions related to the accuracy or integrity of any part of the work are appropriately investigated and resolved.

\section{Funding}

Funders played no role in study design; data collection, analysis, or interpretation; or writing the article. The corresponding author had full access to all data and responsibility for the decision to submit for publication. Open access funding provided by Karolinska Institute.

\section{Competing interests}

The authors declare no competing interests.

\section{Additional information}

Correspondence and requests for materials should be addressed to J.W.

Reprints and permissions information is available at www.nature.com/reprints.

Publisher's note Springer Nature remains neutral with regard to jurisdictional claims in published maps and institutional affiliations. 
(c) (i) Open Access This article is licensed under a Creative Commons Attribution 4.0 International cc) License, which permits use, sharing, adaptation, distribution and reproduction in any medium or format, as long as you give appropriate credit to the original author(s) and the source, provide a link to the Creative Commons licence, and indicate if changes were made. The images or other third party material in this article are included in the article's Creative Commons licence, unless indicated otherwise in a credit line to the material. If material is not included in the article's Creative Commons licence and your intended use is not permitted by statutory regulation or exceeds the permitted use, you will need to obtain permission directly from the copyright holder. To view a copy of this licence, visit http://creativecommons.org/licenses/by/4.0/.

(C) The Author(s) 2021 